Mode Processing Influenced by

Music Training and Language Experience-An ERP Study

Shuqing Zhao, Prof. Dr. Ben Godde

Jacobs University Bremen

May 2019 


\begin{abstract}
Major and minor modes are closely linked to the emotional properties of music. Previous research on the late positive components (LPC) of auditory event-related potentials (ERP) suggests that the minor mode is perceived as a lower-pitched deviant from the major mode. In this study, we compared the behavioral and ERP results of 30 musicians and 29 non-musicians who are either tone- or intonation-language speakers on a mode processing task. Musicians and intonation-language speakers responded with higher accuracy on the mode classification task than their counterparts. Musicians showed an enhanced N1/P2 complex in auditory ERPs, indicating increased sensitivity to pitch. A global field power analysis revealed that intonation-language-speaking musicians had a robust and long-lasting LPC in response to critical notes in both modes, whereas musicians who spoke a tone-language had an attenuated LPC response. Non-musicians didn't have a robust LPC for either mode. Our overall results contradict the theory that major-mode music is perceived as the default mode based on previous musical experience. ERP differences between tone- and intonation-language speakers during mode processing reflect transfer of training effects between musical and language experience, possibly resulting from auditory perceptual learning for common acoustic processing in the two domains. The current findings support a psychophysical instead of enculturational explanation for mode processing.
\end{abstract}

Keywords: mode perception, tone language, auditory, event-related potentials, LPC 


\section{Mode Processing Influenced by \\ Music Training and Language Experience-An ERP Study}

In music theory, major and minor modes are distinct entities with emotional associations. The difference between major and minor modes lies in the different intervals between adjacent notes on a music scale: a minor-mode scale consists of notes with lower pitches than their major-mode counterparts. Major-mode music is usually perceived as happy, and minor mode sad. Similar to tempo, which also influences the emotional properties of music (Dalla Bella, Peretz, Rousseau, \& Gosselin, 2001; Swaminathan \& Schellenberg, 2015), mode can be processed in a spontaneous manner upon listening, as described by previous EEG studies (Bakker \& Martin, 2015; Halpern, Martin, \& Reed, 2008), and is independent of the overarching musical structure (Bostwick, Seror, \& Neill, 2018). In contrast, emotional information conveyed by harmony (Tramo, Cariani, Delgutte, \& Braida, 2001) and timbre (Hailstone et al., 2009) need to be parsed through prolonged listening (Patil, Pressnitzer, Shamma, \& Elhilali, 2012). Nevertheless, unlike the emotional perception from tempo information, whose mechanism is explained to a large extent by biological factors such as arousal, mode perception involves much more complex processes (Parncutt, 2014) and requires further research.

Mode processing is believed to occur naturally when people listen to music as the relational properties of a musical scale is processed automatically in the auditory cortex (Brattico, Tervaniemi, Näätänen, \& Peretz, 2006; Parncutt, 2014). When asked to judge the emotion expressed through music, older children (8 years) make use of both tempo and mode as cues, whereas young children (3-4 years) only base their judgment on tempo (Dalla Bella et al., 2001), implying that mode processing is not an innate ability. This leads to the question as to whether there are individual and cultural differences in mode processing. Existing research on the emotional connotations of musical mode processing have focused on either enculturational or psychophysical explanations (Parncutt, 2014).

The enculturation theory proposes that the major mode is used more commonly in most music genres, and is therefore a "default" for most listeners (Swaminathan \& Schellenberg, 2015). Compared to major-mode music, the pitches in the minor mode are 
perceived as "lower than normal" (Pearce \& Wiggins, 2012) and unfamiliar. Since familiarity is often associated with positive emotions, the unfamiliar minor mode might be perceived as relatively sadder than the major mode (Parncutt, 2014). In addition, music pieces involving negative emotions such as sadness and fear are often composed in minor mode (Laurier, Lartillot, Eerola, \& Toiviainen, 2009), and musical experience as such could further strengthen the perceptual link between mode and emotions.

In support of this theory, an auditory event-related potential (ERP) study revealed a late positive component (LPC) in musicians in response to melodies in the minor mode, but not the major mode; non-musicians did not have a robust LPC for critical notes in either mode (Halpern et al., 2008). The LPC can be observed when expectations for stimuli are violated in an oddball paradigm (Brattico et al., 2006). Since musicians have more exposure to music in both modes, it is believed that the major-minor distinction comes from their relatively more experience with major-mode music.

Based on the psychophysical theory, mode may be among one of the many acoustic properties that can influence emotional perception ( (Dalla Bella et al., 2001; Swaminathan \& Schellenberg, 2015), pitch distribution (Huron, Yim, \& Chordia, 2010), pitch range (Swaminathan \& Schellenberg, 2015), and timbre (Hailstone et al., 2009). Even basic musical triad chords, the most temporally limited representations for mode, are known to retain emotional information: minor triads are associated with negative emotional connotations and major triads are associated with positive emotional connotations (Bakker \& Martin, 2015).

Research has shown that listeners are sensitive to musically expressed emotions in an unfamiliar tonal system, which involves novel scale intervals and therefore unfamiliar modes, suggesting that the emotional information contained in mode is conveyed through the acoustic properties of music, and is not bound to prior experience (Balkwill \& Thompson, 1999). In support of this theory, experiments using artificial scales have found that music is perceived as sadder when the interval between two pitches is reduced arbitrarily (Huron et al., 2010).

Considering the fact that minor-mode music involves lower pitches (Huron et al., 
2010; Parncutt, 2014) and smaller pitch intervals (Huron \& Davis, 2013) than major-mode music, a possible explanation for the universality of mode perception is that the pitch combinations of minor chords resemble those of sad speech, which is independent of any specific language and culture (Cook, Fujisawa, \& Takami, 2006). Musical training has been found to alter the neural responses to speech prosody that convey different emotions, indicating a shared psychoacoustic basis of language and musical emotional processing (Park et al., 2014), such as that in mode perception.

In this study, we will use the auditory ERP paradigm from Halpern et al. (2008) to explore the transfer of training effects between musical and language experience, particularly the effect of speaking a tone-language on mode processing abilities.

Speakers of tone-languages are known to have differential neurophysiological responses to music, compared with speakers of intonation-languages (Bidelman, Gandour, \& Krishnan, 2011; Gandour et al., 2003). The transfer of training effects imply that experience with tonal properties in language might have a similar effect on auditory perception as musical training (Besson, Chobert, \& Marie, 2011). Musicians are found to have better behavioral outcomes on various auditory tasks (Bidelman et al., 2011; Halpern et al., 2008; Magne, Schön, \& Besson, 2006). In addition, the amplitude of N1/P2 components in auditory evoked potentials is enhanced for musicians (Magne et al., 2006; Shahin, Bosnyak, Trainor, \& Roberts, 2003; Tremblay, Ross, Inoue, McClannahan, \& Collet, 2014), compared to non-musicians, which may reflect improved sensitivity to auditory stimuli and implicit pitch discrimination processes (Winkler, Denham, \& Escera, 2013). Nevertheless, non-musicians showed an increase in the N1/P2 complex amplitude after receiving auditory perceptual training with speech stimuli (Tremblay et al., 2014). Tone-language speakers are believed to be more sensitive to tonal properties and local pitch differences in speech, reflected both in neural responses and perception (Alexander, Bradlow, Ashley, \& Wong, 2008; Besson et al., 2011). However, other studies have found that tone-language speakers only have enhanced neural responses during musical tasks and do not show perceptual benefits (Bidelman et al., 2011; Stevens, Keller, \& Tyler, 2013).

In the current study, we extended the auditory ERP study of Halpern et al. (2008) to 
the comparative linguistic domain. By drawing a comparison among musicians and non-musicians who are either tone- or intonation-language speakers, we would be able to explore the influence of language and musical experience on auditory perception, especially on mode processing.

There have been studies that relate mode processing to either cultural experience or music training, but not both, and the culture variable is often hard to determine. Previous studies have revealed that mode can be perceived independently of culture and musical experience (Balkwill \& Thompson, 1999), but researchers did not agree on whether mode processing is an enculturated or purely psychophysical process (Parncutt, 2014). Findings from the current study could add evidence to the debate, thereby shedding light on the question as to whether emotional perception in music is universal or culture-specific.

Since language is an interface between culture and auditory perception, studying the transfer of training effects from language experience to music perception could be an alternative approach to disentangle auditory perceptual experience and the enculturation effect. Namely, if tone- and intonation-language speakers with a similar level of music training who likely come from different cultural backgrounds show comparable outcomes on a mode classification task, and if experience with tonal properties in language has a similar influence on mode processing to musical experience, we might be able to infer that the modality-based emotional processing in music is more or less universal.

We hypothesized that tone-language speakers would have neural and behavioral outcomes similar to those of musicians. More specifically, we expected musicians and tone-language speakers to have a higher correct response rate on the major-minor melody classification task and increased N1/P2 amplitudes in the auditory ERPs.

In addition, based on Halpern et al. (2008), we hypothesized that an LPC in response to minor but not major mode-critical notes would be observed in musicians and tone-language speakers, and no LPC would be found in intonation-language-speaking non-musicians.

We also explored potential lateralization patterns for mode processing in tone- and intonation-language-speaking participants. There is currently little research on the 
lateralization of mode processing. Nevertheless, a right lateralization has been found for music listening in general (Santosa, Hong, \& Hong, 2014). In Halpern et al. (2008), the LPC is lateralized to the right hemisphere in two-thirds of the musician participants, though the results weren't statistically significant.

An fMRI study (Gandour et al., 2003) found that tone-language speakers process tone-related pitch contours (local, syllable-based) in the left hemisphere, but intonation-related pitch contours (global, sentence-based) mainly in the right hemisphere; intonation-language speakers were found to process both types of pitch contours in the right hemisphere. Even though the study used speech-like stimuli, the tone-related pitch contours are closely related to the critical notes that determine mode in music, whereas the intonation-related pitch contours resemble melody (Vaissière, 1983). A potential reason for this differentiation is the neurobiological adaptation effect for pitch processing: tone-language speakers might have a functional differentiation between local and global

pitch processing in speech, due to their experience with and perceptual need for fine tonal contrasts.

We therefore hypothesized that mode processing, which can be observed as a differential LPC response to mode-critical notes, might be lateralized to the left hemisphere for tone-language speakers. We hypothesized a right lateralization of mode processing for intonation-language speakers, similar to the lateralization pattern of general musical perception.

\section{Methods}

\section{Participants}

We recruited 70 participants from the undergraduate and graduate students at Jacobs University Bremen. All participants were right-handed. None of them had a history of neuropsychiatric conditions or hearing disorders. All participants gave informed consent before participating and received either bonus points for psychology and statistics courses or cafeteria credit as compensation. 7 participants were excluded from the study because of high impedance level prior to EEG recording caused by particularly thick and full 
hairstyle. 4 other participants were excluded due to inconsistent answers regarding their musical and language experience.

59 participants were included in the final study and the data analyses that follow. The participants were divided into 4 groups: 1) intonation-language-speaking musicians $(\mathrm{N}=15) ; 2)$ intonation-language-speaking non-musicians $(\mathrm{N}=15) ; 3)$ tone-language-speaking musicians $(\mathrm{N}=15)$; and 4) tone-language-speaking non-musicians $(\mathrm{N}=14)$. Table 1 displays the information on musical and language experience of all participants included in the analysis.

Language Experience. The participants' language experience was assessed using self-report measures, i.e., their native language(s), other language(s) they know, and the proficiency level of each language. The responses were later examined by the experimenter and recoded as either tonal and intonation languages. Since we hypothesized an auditory perceptual effect of tonal language experience, oral communication was prioritized when determining fluency in a certain language, rather than written communication. The languages from the responses of all qualified participants can be found in Appendix B, as well as their categorization as either tonal or intonation.

On average, our participants spoke a total of 3 to 4 languages, including 1 to 2 that they know as native languages, and 2 to 3 languages that they speak fluently. We defined any language that the participants learned before 1 year of age as a native language, as previous research reveals that the abilities to detect tonal properties in languages deteriorate before 2 years of age (Liu \& Kager, 2018). To control for the language effect, we ensured that none of the participants in the intonation language groups spoke a tonal language or any pitch-accent language, which contains limited tonal properties (e.g., Japanese, Serbian).

Musical Experience. The participants' musical experience was assessed using items from the Music USE (MUSE) Questionnaire (Chin \& Rickard, 2012). The amount of musical experience was hypothesized to have a critical influence on the LPC response to major or minor tunes. Since it is unknown whether the amount of musical listening has a similar effect as musical practice, we considered both musical listening (Index of Music 
Table 1

Descriptive Statistics of Participant Information

\begin{tabular}{|c|c|c|c|c|c|c|c|c|c|c|}
\hline & \multirow[t]{2}{*}{ Language Groups } & \multirow[t]{2}{*}{$\mathrm{N}$} & \multirow[t]{2}{*}{ Age } & \multicolumn{4}{|c|}{ Language Experience (No.) } & \multicolumn{3}{|c|}{ Musical Experience } \\
\hline & & & & Total & Native & Intonation & Tonal & Years of Practice & IML & IMIP \\
\hline \multirow[t]{2}{*}{ Musician Groups } & \multirow[t]{2}{*}{ Intonation } & \multirow[t]{2}{*}{15} & 19.530 & 3.667 & 1.267 & 2.200 & 0 & 11.670 & 12.870 & 21.365 \\
\hline & & & $(0.990)$ & $(0.976)$ & $(0.458)$ & $(0.941)$ & (0) & $(3.069)$ & $(5.330)$ & $(20.458)$ \\
\hline \multirow{6}{*}{ Non-musician Groups } & \multirow{2}{*}{ Tonal } & \multirow{2}{*}{15} & 20.640 & 3.000 & 1.133 & 1.067 & 1.133 & 11.270 & 12.530 & 16.863 \\
\hline & & & $(2.170)$ & $(0.845)$ & $(0.352)$ & $(0.258)$ & $(0.352)$ & $(3.327)$ & $(6.823)$ & (19.554) \\
\hline & \multirow{2}{*}{ Intonation } & \multirow{2}{*}{15} & 20.600 & 3.467 & 1.200 & 2.400 & 0 & 0.081 & 11.130 & 0.004 \\
\hline & & & $(2.772)$ & $(0.640)$ & $(0.414)$ & $(0.632)$ & (0) & $(0.260)$ & $(7.289)$ & $(0.010)$ \\
\hline & \multirow{2}{*}{ Tonal } & \multirow{2}{*}{14} & 22.000 & 3.214 & 1.643 & 1.500 & 1 & 0.089 & 13.070 & 0.011 \\
\hline & & & (3.328) & (1.311) & $(0.928)$ & $(0.650)$ & (0) & $(0.264)$ & $(7.416)$ & $(0.033)$ \\
\hline \multirow{2}{*}{\multicolumn{2}{|c|}{ Total }} & \multirow{2}{*}{59} & 20.670 & 3.339 & 1.305 & 1.797 & 0.525 & 5.872 & 12.390 & 9.722 \\
\hline & & & (2.550) & $(0.976)$ & (0.595) & $(0.846)$ & $(0.568)$ & (6.159) & $(6.620)$ & (16.992) \\
\hline
\end{tabular}

Note: Description of participants' language and musical experience. The language experience columns display the average number of the language(s) participants know, of their native language(s), and of intonation or tone language(s) they are fluent in. IML: Index of Music Listening (minimum $=1$, maximum $=25$ ). IMIP: Index of Music Instrument Playing $($ minimum $=0)$.

Listening, IML) and musical practice (Index of Music Instrument Playing, IMIP).

IML (minimum possible score $=1$, maximum possible score $=25$ ) was calculated based on the frequency and duration of music listening. IMIP (minimum possible score $=$ 0) was calculated based on the years of instrument playing or singing experience, duration of practice, and regularity of practice. There is no significant difference on IML between the four groups, indicating a comparable amount of music listening by all groups. Tonal language-speaking musicians have 11.27 years of musical experience on average, and intonation language-speaking musicians 11.67 years. All participants in the non-musician groups had no more than 6 months of musical experience. There is no significant difference on IMIP and the years of musical practice between the two musician groups or between the two non-musician groups. 


\section{Materials}

Melody listening task. For the major-minor melody classification task, we used the stimuli from Halpern et al. (2008). In total, 48 melody stimuli were synthesized. Half of the melodies were generated from existing melodies, and each was modified to create an other-modality twin, differing from the original only on the critical notes. 19 out of 24 of the original melodies were in major mode, and 5 were in minor mode. On average, the tunes had a duration of $8.425 \mathrm{~s}(\mathrm{SD}=1.689 \mathrm{~s})$, and the critical note occurred at $1.071 \mathrm{~s}$ $(\mathrm{SD}=0.571 \mathrm{~s})$ after the melody onset. The melodies had 2.387 note counts per second on average ( $\mathrm{SD}=0.447)$. Of the 24 tune pairs, 11 were in C Major/Minor, 5 in D Major/Minor,and the rest were in the in keys of E, F, G, and A. Two-thirds of the tunes began on the tonic and the rest on the dominant of the scale (Halpern et al., 2008). While the melodies differed on various measures such as tempo, contour, and length, each major-minor pair was matched in all parameters except for mode. Therefore, it is meaningful to compare the responses to major and minor melodies despite the variance on other parameters among the stimuli.

Chord listening task. To better assess the perception of musical mode across all four groups, as well as to segregate the effects of pitch and mode on the emotional perception of music, we prepared 48 triads (24 major, 24 minor) whose root notes ranged from F3 $(174.61 \mathrm{~Hz})$ to E5 (659.25 Hz), i.e., spanning exactly 2 octaves on the well-tempered scale. The triads were generated using the piano part from MuseScore, trimmed to $800 \mathrm{~ms}$, and were normalized to the same loudness.

\section{Procedure}

Before the experiment, participants given information on the study and asked for their informed consent. Then, all participants were asked to fill out a screening questionnaire on their handedness, hearing abilities, and neuropsychological health, as well as a questionnaire regarding their musical and language experience. Participants who did not meet the criteria were excluded from further participation.

The auditory stimuli were presented at a comfortable volume level (approximately 
$55 \mathrm{~dB}$ ) using the frontal, monaural built-in loudspeaker of a Philips computer. The participants were seated about $50 \mathrm{~cm}$ away from the monitor in a comfortable chair.

Mode classification task. To measure the participant's perceptual abilities for mode and record neural signals from a mode processing task, we used the paradigm from Halpern et al. (2008), where the participant is asked to categorize major and minor melodies as either "happy" or "sad". There is evidence that emotional labels can serve as cues on the major-minor mode-classification task: the major mode is perceived as happy and the minor mode as sad. While only musicians are able to label music as "major" or "minor", even people without music training can perform with relatively high accuracy on the mode-classification task when instructed to evaluate the emotional value expressed in music (Halpern et al., 2008).

At the beginning of the task, participants were given examples of one major and one minor melody, described as having a "happy" and "sad" emotional color based on composers' intention, respectively. Following the examples, they were provided with 4 training trials, where they had to make a judgment of either "happy" or "sad" about the music they've heard by pressing a custom-built button, and were given feedback for their response ("correct" or "incorrect"). The correct response linked with major and minor melodies were "happy" and "sad", respectively.

During the task, the participant was presented with 24 major and 24 minor melodies, i.e., 24 melody pairs, and was instructed to respond by making emotional judgments in the same way as in the practice task. However, no feedback was given on the real task. Each stimulus was presented twice in the experiment. Each stimulus followed an interstimulus interval (ISI) of either 1,000 or $1,500 \mathrm{~ms}$, which was randomized for each trial. During the ISI, the participant was instructed to prepare for the melody and blink if they wished. While the melody was playing, the participant was instructed to refrain from blinking or moving their eyes as much as they could. After listening to each melody, the participant had to make an affective judgment of the tune.

We randomized the trials in a way that ensured each of the 48 melody was presented once before any melody was repeated. The major and minor melody in a pair was 
separated from each other by exactly 24 trials. The experiment was divided into 8 blocks of 12 melodies each. There was a self-paced break after every block. The classification task lasted about $20 \mathrm{~min}$.

Chord rating task. After the melody listening task and a self-paced break, the participants were presented with 48 triads (24 major, 24 minor) in a random order without repetition. After listening to each triad, the participant was presented with a question ("How does the chord sound?") and response options on a 4-point Likert scale $(1=$ very sad, 4 = very happy). They were asked to respond by pressing one of the four buttons with their right index finger. EEG was not recorded during this task.

\section{EEG Recordings}

EEG data were recorded using a 32-channel active electrode system (ActiveTwo, BioSemi, Amsterdam, Netherlands). 32 electrodes were positioned using elastic caps in accordance with the International 10-20 system (Jasper, 1958). Two external electrodes were positioned above and below the right eye for ocular artifact detection. During the task, EEG signal was recorded with a sampling rate of $2048 \mathrm{~Hz}$ and online band-pass filtered between 0.16 and $100 \mathrm{~Hz}$.

\section{EEG Data Analysis}

The EEG recordings were imported into MATLAB (The MathWorks, Inc., 2019) and offline analyzed using the toolboxes EEGLab (Delorme \& Makeig, 2004) and ERPLab (Lopez-Calderon \& Luck, 2014).

Data was first downsampled to $512 \mathrm{~Hz}$ and mapped onto a DIPFIT spherical model. A notch filtered of $50 \mathrm{~Hz}$, a high-pass filter of $0.5 \mathrm{~Hz}$, and a low-pass filtered of $30 \mathrm{~Hz}$ were applied sequentially. Scalp electrode channels with abnormal activity were detected based on normalized kurtosis, and channels with an absolute $z$-score of 5 or higher were rejected. The removed channels were then interpolated using the spherical method. An independent component analysis (ICA) was used to identify artifact components for later removal. However, the quality of the ICA varied with the number of interpolated channels in each dataset. We therefore decided to disregard the ICA results. 
Bin-based epochs of major and minor tunes were extracted relative to the critical note, ranging from $300 \mathrm{~ms}$ prestimulus interval to 2,000 $\mathrm{ms}$ after the onset of the critical note. Epochs were baseline corrected relative to the prestimulus interval and lowpass filtered at $20 \mathrm{~Hz}$.

An automatic artefact detection was carried out for both scalp and external channels using the moving window peak-to-peak threshold method in ERPLAB (window width $=$ $200 \mathrm{~ms}$, window step $=100 \mathrm{~ms}$ ). Epochs with a peak-to-peak voltage difference exceeding $100 \mu \mathrm{V}$ were marked for rejection. Next, epochs containing step-like artifacts (threshold = $15 \mu \mathrm{V}$ ) were marked using the step-like artifacts test in ERPLAB (window width $=400 \mathrm{~ms}$, window step $=10 \mathrm{~ms}$ ). Epochs marked for rejection were plotted after the automatic artifact detection process. Based on visual inspection, the automatic methods resulted in a high number of misclassified epochs. We therefore disregarded the automatic artifact detection results. The following results are based on all extracted epochs (major tunes: $\mathrm{N}$ $=48$; minor tunes: $\mathrm{N}=48 ; 59$ participants).

\section{Results}

\section{Behavioral Results}

Chord ratings. Statistical analyses of the data were carried out in $\mathrm{R}$ ( $\mathrm{R}$ Core Team, 2019). Using the lme4 package (Bates, Maechler, Bolker, \& Walker, 2015), we conducted a mixed-design repeated-measures analysis of variance (ANOVA) to examine the mode processing abilities of the four groups. There were significant main effects of the musical experience of the participant, $F(1,2772)=14.78, p<.001, r=.07$, and the mode of the chord, $F(1,2772)=426.77, p<.001, r=.36$. The ratings from tonal and intonation language speakers did not differ in general, $F(1,2772)<1, r=.01$, and there was no significant interaction effect between participants' musical and language experience, $F(1$, 2772) $<1, r=.01$.

Figure 1 displays the ratings for major and minor chords over a pitch range of F3 $(174.61 \mathrm{~Hz})$ to E5 $(659.25 \mathrm{~Hz})$, averaged across each group. All four groups exhibited a clear linear trend between their ratings and pitch. As shown in the top left and bottom left 

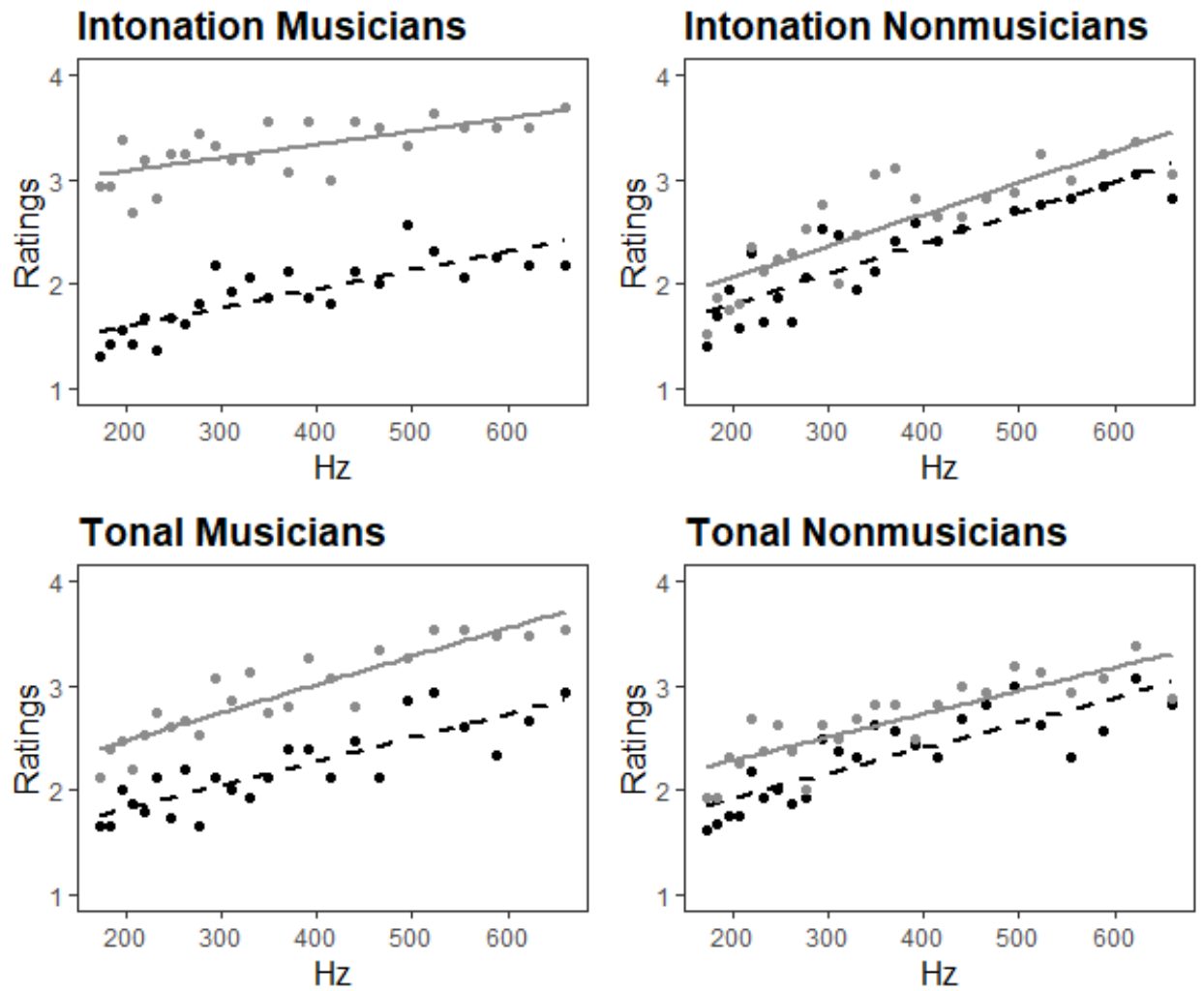

Figure 1. Affective ratings for major and minor triads across all music and language groups. $x$-axis: fundamental frequency of the root note of the triad. $y$-axis: participant rating averaged across each group ( $1=$ very sad, $4=$ very happy). The data points in grey represent mean ratings of major triads, and the data points in black represent mean ratings of minor triads. The solid and dashed lines show the linear trend between pitch and rating of major and minor triads, respectively.

panels, musicians on average discerned major and minor chords more distinctly than non-musicians. Notably, intonation-language-speaking musicians gave more distinct ratings based on mode than tone-language-speaking musicians, who discerned more between lower and higher pitches. Compared to musicians, non-musicians were equally capable of judging the valence of chords based on pitch, but not mode.

To better understand whether pitch influenced mode perception in each group of participants, we computed the difference between the ratings (Major - Minor) at every pitch level. For each of the four groups, a linear regression was conducted between the rating difference and pitch (expressed as the fundamental frequency of the root note of the triad in Hz). As shown in Table 2, a significant regression equation was found only for tone 
Table 2

Linear Regression Results (Pitch as Independent Variable)

Dependent variable: rating difference

\begin{tabular}{lcccc} 
& \multicolumn{2}{c}{ Intonation } & \multicolumn{2}{c}{ Tone } \\
& Musicians & Non-musicians & Musicians & Non-musicians \\
\hline Pitch & -0.001 & -0.0001 & 0.0004 & $-0.001^{* * *}$ \\
& $(0.0004)$ & $(0.0004)$ & $(0.0004)$ & $(0.0002)$ \\
Constant & $1.539^{* * *}$ & $0.309^{*}$ & $0.568^{* * *}$ & $0.765^{* * *}$ \\
& $(0.142)$ & $(0.157)$ & $(0.147)$ & $(0.098)$ \\
$R^{2}$ & 0.087 & 0.006 & 0.055 & 0.362 \\
Adjusted $R^{2}$ & 0.045 & -0.039 & 0.012 & 0.333 \\
Residual Std. Error $(\mathrm{df}=22)$ & 0.255 & 0.282 & 0.263 & 0.175 \\
$F(1,22)$ & 2.091 & 0.140 & 1.282 & $12.497^{* * *}$ \\
\hline
\end{tabular}

Note:

${ }^{*} \mathrm{p}<0.1 ;{ }^{* *} \mathrm{p}<0.05 ;{ }^{* * *} \mathrm{p}<0.01$

language-speaking non-musicians $(F(1,24)=12.497, p<.01)$, with an $R^{2}$ of .362. The emotional ratings of tone-language-speaking musicians for the triads converged at the higher-pitch end, i.e., they might disregard mode and perceive all higher-pitched chords as happier-sounding. On the other hand, for tone-language-speaking musicians, a positive but not significant relationship was found between the rating difference and pitch, implying that they might discriminate mode better with higher-pitched rather than lower-pitched triads.

Mode classification accuracy. An ANOVA comparing the four music and language groups revealed significant main effects of musical and language experience on the correct response rate: musicians performed significantly better than non-musicians $(F(1,124)=$ 51.434, $p<.0001$ ) and intonation-language speakers performed significantly better than tone-language speakers $(F(1,124)=4.455, p<.05)$. There is no significant interaction effect of the language and musical experience of the participants $(F(1,124)=0.013, p=$ 
.908). The Tukey HSD test revealed that there was no significant difference between intonation- and tone-language-speaking musicians $(p=.328)$ or non-musicians $(p=.392)$. Figure 2 displays the overall correct response rate of each group on the melody listening task.
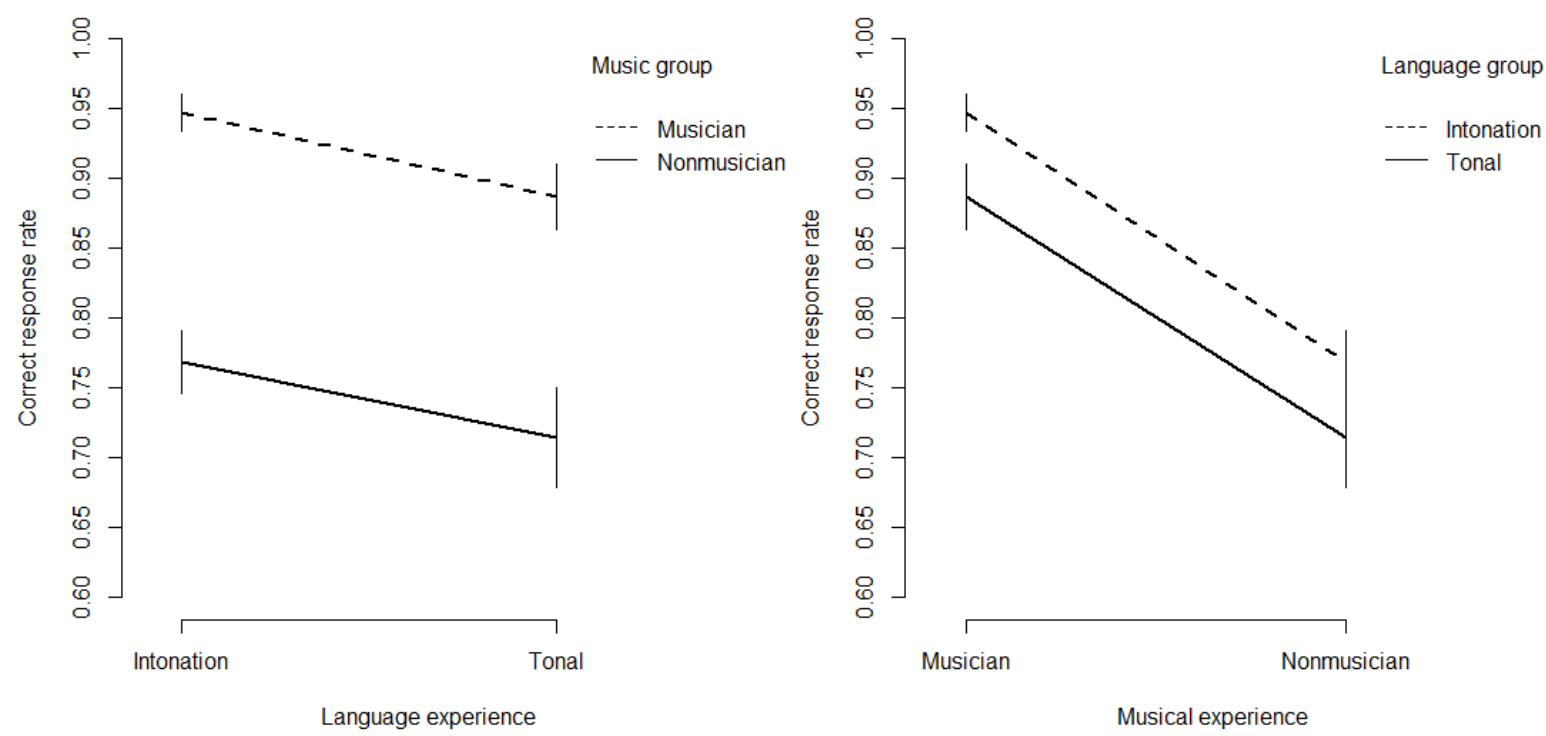

Figure 2. Interaction plots of the behavioral results from the melody listening task. y-axis: correct response rate on the task. The vertical bars represent the standard error of the mean of the respective group. As shown on the left, musicians have a much higher correct response rate than non-musicians, regardless of their language experience. As shown on the right, the two intonation-language speaking groups had higher correct response rates than their tonal-language-speaking counterparts with a similar level of musical experience.

\section{EEG Results}

Figure 3 displays the group-averaged ERP waveforms evoked to major and minor tunes in several scalp regions. To explore whether there is a lateralization pattern for mode processing, we averaged the channel data of left and right frontal, temporal, and parietal regions (e.g., $\mathrm{LF}=\mathrm{F} 3$ and $\mathrm{F} 7$ ).

While all groups showed N1/P2 components to the onset of the critical note, as can be seen over frontal and parietal electrode sites, musicians had a much larger N1/P2 response, especially over frontal and parietal regions in particular, as compared to 


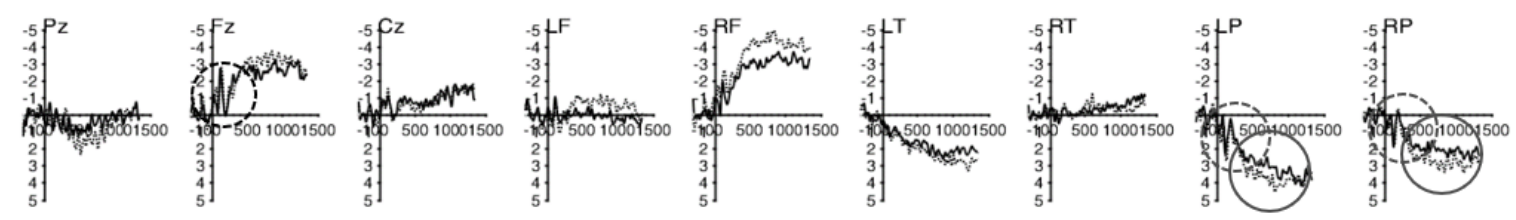

(A) Intonation language-speaking musicians
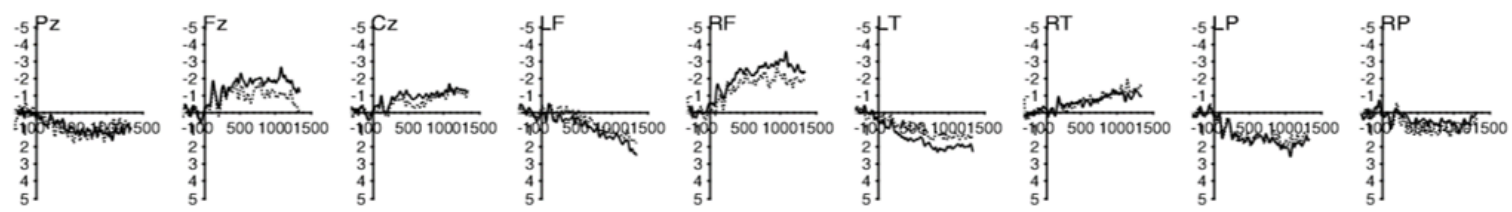

(B) Intonation language-speaking non-musicians
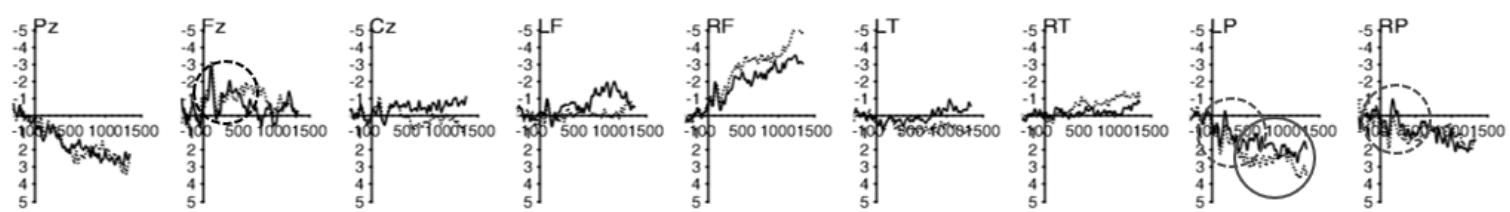

(C) Tone language-speaking musicians
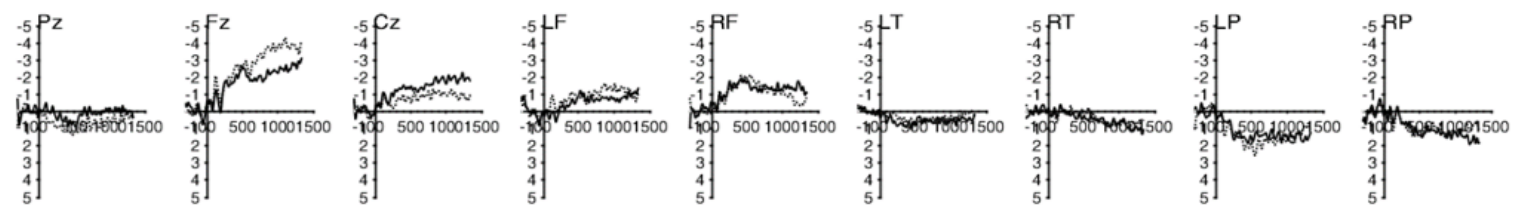

(D) Tone language-speaking non-musicians

Figure 3. Group-averaged ERP waveforms evoked by major (solid lines) and minor (dashed lines) tunes. $x$-axis: time relative to critical note onset (ms). $y$-axis: amplitude $(\mu \mathrm{V})$. The waveforms in columns 4 to 9 of each row was constructed by averaging the data at two electrodes located over a scalp region $(\mathrm{LF}=\mathrm{F} 3$ and $\mathrm{F} 7 ; \mathrm{RF}=\mathrm{F} 4$ and $\mathrm{F} 8$; $\mathrm{LT}=\mathrm{T} 7$ and C3; $\mathrm{RT}=\mathrm{T} 8$ and C4; LP $=\mathrm{P} 3$ and P7; RP $=\mathrm{P} 4$ and P8). All groups showed an N1/P2 component to the onset of the critical note over Fz. The N1/P2 component was enhanced for musicians over frontal and parietal regions (indicated by dashed circles), as compared to non-musicians. Only intonation-language-speaking musicians showed a prominent LPC over both parietal electrode sites (indicated by solid circles). Tone-language-speaking musicians had an LPC over left parietal region only. For every group and electrode site, the LPC amplitude evoked by minor tunes was larger than that to major tunes. 
non-musicians, as shown in Figure 3. Intonation language-speaking musicians had a prominent LPC over both left and right parietal regions, whereas tone language-speaking musicians only showed a LPC over the left parietal region. While both major and minor tunes elicited late positive components in musicians, for both groups and every scalp region, the LPC amplitude evoked by minor tunes was larger than that evoked by major tunes. Contrary to our hypothesis, there was no clear lateralization pattern for the LPC evoked by mode-critical notes. Figure 4 displays the mean global field power (GFP) waveforms generated from group-averaged ERPs evoked by major and minor tunes. GFP is the standard deviation of the potentials at all electrodes of an average-reference map. It is useful for identifying moments of high signal-to-noise ratio, corresponding to moments of high global neuronal synchronization, and can be used to interpret overall ERP components in multichannel EEG data (Brunet, Murray, \& Michel, 2011; Hamburger \& v.d. Burgt, 1991; Skrandies, 1990). As shown in Figure 4, the N1/P2 component is enhanced for musicians, as compared to non-musicians. However, only intonation-language-speaking musicians show a robust and long-lasting late positive component starting around $400 \mathrm{~ms}$. In contrast, while tone-language-speaking musicians have a relatively more robust LPC response after around $300 \mathrm{~ms}$ than both non-musician groups, this activity was not as strong as the LPC response of musicians who spoke intonation language(s) only.

\section{Discussion}

Results from the current study are in line with research that shows musicians are more sensitive to musical properties such as mode and pitch. Compared with non-musicians, musicians perceived the emotional qualities of major and minor triads as much more distinct, as shown in Figure 1. In the major-minor melody classification task, musicians also performed with significantly higher accuracy than non-musicians. In addition, musicians had enhanced neural responses to critical notes in major and minor melodies, as seen in the increased N1/P2 component amplitude and a robust late positive component in parietal regions.

In contrast to Halpern et al. (2008), we did not find a differential LPC response to 


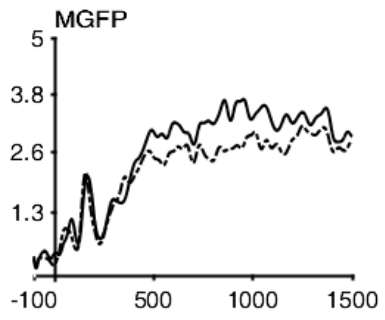

(A)

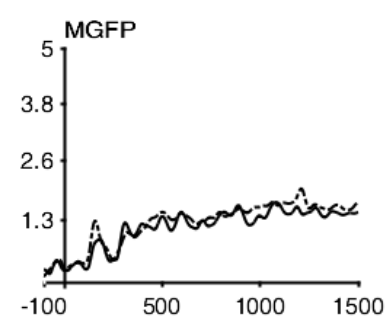

(B)

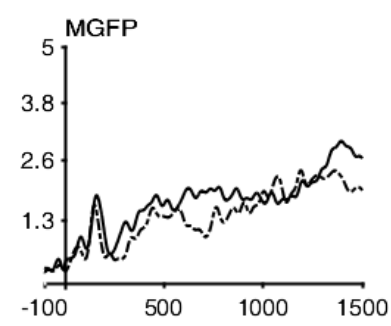

(C)

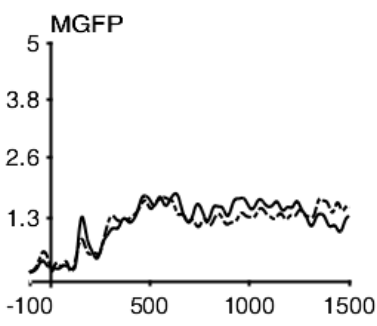

(D)

Figure 4. Mean global field power generated from group-averaged ERP waveforms evoked by major (solid lines) and minor (dashed lines) tunes. $x$-axis: time relative to critical note onset (ms). $y$-axis: amplitude ( $\mu \mathrm{V})$. A: intonation-language-speaking musicians; B: intonation language-speaking non-musicians; C: tone-language-speaking musicians; D: tone-language-speaking non-musicians. The N1/P2 component is enhanced for musicians. Only intonation-language-speaking musicians show a robust and long-lasting late positive component starting around $400 \mathrm{~ms}$.

major and minor melodies in musicians. Both major and minor melodies elicited LPC responses in musicians and not in non-musicians, thought the LPC responses to minor melodies had a higher amplitude than that to major melodies. This finding challenges the view that the minor mode is perceived as "abnormal" or "deviant" based on auditory perceptual learning during participants' prior musical experience. These results also raised the question of the underlying mechanisms for LPC in auditory ERPs. The LPC is commonly considered a marker for cognitive classification processes, especially when experiencing an unusual stimulus in the oddball paradigm (Brattico et al., 2006). However, the implication of LPC responses in auditory paradigms might need further investigation.

Curiously, intonation language-speaking musicians displayed a much stronger LPC response to critical notes than tone-language-speaking musicians. As shown in Figure 4 , the GFP waveform of tone language-speaking musicians is more similar to that of tone language-speaking non-musicians, rather than to that of intonation language-speaking musicians. However, the behavioral results indicate that musicians and non-musicians have comparable mode processing abilities, regardless of their language experience. This mismatch between behavioral and electrophysiological findings implies a complex 
relationship between auditory perceptual learning from musical and language experience, particularly between the tonal properties of languages and musical mode processing.

Previous research suggests that attention might be an important confounding factor influencing the amplitude of auditory ERP components on auditory perceptual tasks (Polich, 1986). Treder, Purwins, Miklody, Sturm, and Blankertz (2014) found that attended deviants but not unattended deviants in an auditory oddball paradigm led to a robust P300 component in the ERPs. Background EEG variation, such as the ultradian rhythm, might also contribute to the individual variability of the P300 component (Polich, 1997). Participants with more interest on the task might therefore be more attentive and have increased ERP amplitudes.

In addition, our participants come from a diverse language background. Considering that $50 \%$ of the world population is at least bilingual (Grosjean, 2001) and more than 60\% of all world languages are tonal (Yip, 2002), our sample is a valid reflection of the actual linguistic environment. However, this also makes it more difficult to interpret results on language experience. While we hypothesized that tone language-speaking participants might have an advantage in mode processing, they turned out to have a lower correct response rate on the major-minor melody categorization task, indicating that they might in fact be less capable of processing mode. In different tone languages, the total number of semantically-significant tones, as well as the acoustic properties of existing tones often differ. Participants who are exposed to tone languages that involve subtler tonal characteristics might therefore be more sensitive to auditory stimuli than those who speak tone languages that have impoverished tonal properties.

Another possible explanation for the underperformance of tone-language-speaking participants on the major-minor mode classification task is that they have a higher sensitivity to pitch, which varies across the melody and might overshadow the emotional properties indicated by mode. As shown in the results of the chord rating task, for tone-language speakers, particularly those without musical experience, the ability to make emotional judgments based on mode was influenced heavily by pitch. The overall pitch range of the melody contour, as well as variance of pitch within the melody, might therefore 
influence the emotional perception of tone-language speakers more strongly than that of intonation-language speakers. On the other hand, this finding is in line with previous research that suggest tone-language speakers only have enhanced neural responses to acoustical properties, but not perceptual ones (Bidelman et al., 2011; Stevens et al., 2013).

Since language and music both require auditory input, language experience might influence the neural mechanisms of audition in a similar way as musical experience, but its effect on musical perception could be limited or even counteractive. If this is the case, then the reversed transfer of training effect between language experience and musical perception in the current study, namely that tone-language speakers who should be more sensitive to pitch were in fact less sensitive to musical mode, would suggest shared auditory perceptual learning for acoustic processing in the language and music domains. This would provide a psychophysical explanation for the mechanisms of mode processing.

\section{Limitations}

One limitation to this study is a relatively small sample size and a high degree of variance in musical and language experience of the participants. While the mean amount of musical experience differed significantly between musician and non-musician groups, there also exists much individual difference within the musician groups in that some individuals received much more musical training than others. Even though the descriptive statistics revealed no significant difference in musical experience between the intonation-language-speaking and tone-language-speaking groups, individual factors might have contributed to the differential behavioral and neural results. It is possible that the relationship between musical experience and mode processing abilities is non-linear, i.e., one might need to receive a certain amount of musical input, in order for their mode processing skills to develop. If this is the case, then a similar degree of musical experience among all the musician participants would be desirable, in order to compare intonationand tone-language speakers.

During the melody listening task, participants were instructed to direct their gaze to a fixed point on the screen when the melody was played. A number of participants 
reported after the experiment that they experienced fatigue and eye tiredness because of this instruction. In addition, several participants reported that the varying ISI made it hard to predict when they should be prepared for the next melody to begin. Due to this wrong judgment of resting period duration, the participants might have accidentally blinked more during the initial few notes, which often coincided with the critical note onset, leading to more eye movement artifacts and a lower signal-to-noise ratio. Conducting the EEG recording in the eyes-closed condition might therefore be a useful way to reduce electroocular artifacts, provided that attention on the task can be ensured. Previous research has shown that eyes-open and eyes-closed conditions do not lead to different ERP results for auditory paradigms, especially when examining later positive components such as P300 amplitude and latency (Polich, 1986). However, when eyes were closed, the association between EEG and P300 was disrupted, perhaps because the overall power produced greater EEG variability (Polich, 1997).

Furthermore, there is room for improvement in the preprocessing procedures. After experimenting with several preprocessing methods and sequences, we decided to include all epochs in the analysis to achieve a favorable amount of data points, while potentially reducing the signal-to-noise ratio. While the ERP waveforms from such procedures yielded acceptable results based on visual inspection, it would be better to include a more meticulous artifact rejection protocol, in order to provide cleaner data and a sound qualitative basis for any statistical testing.

Since the automatic artifact detection procedures in ERPLAB resulted in many inaccurately categorized epochs, a manual inspection of the epoch quality would be preferred. We noticed that conducting an ICA after rejecting bad channels and interpolating the rejected channels based on a spherical method resulted in too many lost ranks in the ICA, leading to highly homogeneous ICA components. The reduced ICA quality due to spherical interpolation couldn't be compensated by the increase in signal-to-noise ratio. It is therefore favorable to conduct the ICA on all remaining channels after rejecting bad channels and before spherical interpolation. In addition, running the ICA before epoching turned out to be a highly time-consuming and computationally expensive process. 
Therefore, rejecting bad channels, extracting epochs, running the ICA and rejecting artifactual components, and eventually interpolating the missing channels might be the overall optimal preprocessing procedure.

\section{Future Steps and Implications}

Future research should also examine the underlying mechanisms of the LPC in auditory ERPs. Previous studies have found that attention, respond mode, and task demand are three important factors that determine the amplitude and latency of later positive components such as the P300 (Polich, 1986, 1987).

We're planning on refining the proprocessing procedure using collected data. More specifically, we will reject the bad channels based on automatic detection methods, extract the epochs, run the ICA on 32 electrode channels and reject the artifactual components, and interpolate the missing channels in the end. We expect this procedure to be a good measure for identifying artifacts, as well as for providing a good signal-to-noise ratio. EEG provides excellent temporal resolution on the millisecond level. As many musical features are entrained to time, EEG can be a useful tool for musical information retrieval and auditory brain-computer interface technologies.

Common strategies of retrieving musical information with EEG include analyzing time-averaged event-related potentials and classifying single-trial EEG responses. In various ERP studies, researchers have managed to extract musical features such as rhythm (Stober, Cameron, \& Grahn, 2014), mode (Halpern et al., 2008), tempo (Losorelli, Nguyen, Dmochowski, \& Kaneshiro, 2017), and emotion (Bakker \& Martin, 2015). With an oddball paradigm, it is also possible to infer complex information that can't be retrieved directly, such as segregating a melody stream from polyphonic music (Treder et al., 2014).

With the on-going development in hardware and neural data analysis, we might one day be able to record and decode neural signals with a much higher degree of freedom, which would enable the extraction of perceived auditory information from neural signals. Technologies such as auditory brain-computer interfaces could shed light on alternative forms of communication, and might bring hope to locked-in syndrome patients. Of course, 
this would require a much deeper understanding of the human auditory system, as well as an enormous amount of high-quality neural data.

\section{Conclusion}

In this study, we compared the behavioral and ERP results of musicians and non-musicians who are either tone- or intonation-language speakers on a mode processing task. Our hypothesis that musicians would have enhanced behavioral and neural outcomes in musical mode perception was confirmed. Musicians and intonation-language speakers responded with higher accuracy on the mode classification task than their counterparts. Musicians showed enhanced N1/P2 components in auditory ERPs, indicating increased sensitivity to pitch.

Halpern et al. (2008) found a differential LPC response to major and minor melodies in musicians, which implied that minor mode is perceived as a deviant. However, we found that intonation-language-speaking musicians had a robust and long-lasting LPC in response to critical notes in both modes, whereas tone-language-speaking musicians had an attenuated LPC response and non-musicians didn't have a robust LPC for either mode. In addition, tone language-speaking participants were less sensitive to mode when giving emotional ratings for triads, and based their judgment more on pitch. Altogether, the results of tone-language speakers suggested a more complicated neural functioning for the perception of mode that is not based on prior musical experience.

Our overall results contradict the findings of Halpern et al. (2008). ERP differences between tone- and intonation-language speakers reflect transfer of training effects between musical and language experience. The current findings support a psychophysical instead of enculturational explanation for mode processing. 


\section{References}

Alexander, J. A., Bradlow, A. R., Ashley, R. D., \& Wong, P. C. M. (2008). Music melody perception in tone-language- and nontone-language speakers. The Journal of the Acoustical Society of America, 124, 2495. https://doi .org/10.1121/1.4782815.

Bakker, D. R., \& Martin, F. H. (2015). Musical chords and emotion: major and minor triads are processed for emotion. Cognitive, Affective, \& Behavioral Neuroscience, 15, 15-31. https://doi.org/10.3758/s13415-014-0309-4.

Balkwill, L.-L., \& Thompson, W. F. (1999). A cross-cultural investigation of the perception of emotion in music: Psychophysical and cultural cues. Music Perception: An Interdisciplinary Journal, 17, 43-64. https://doi .org/10.2307/40285811.

Bates, D., Maechler, M., Bolker, B., \& Walker, S. (2015). Fitting linear mixed-effects models using lme4. Journal of Statistical Software, 67, 1-48. https://doi.org/10.18637/jss.v067.i01.

Besson, M., Chobert, J., \& Marie, C. (2011). Transfer of training between music and speech: Common processing, attention, and memory. Frontiers in Psychology, 2, 94. https://doi.org/10.3389/fpsyg.2011.00094.

Bidelman, G. M., Gandour, J. T., \& Krishnan, A. (2011). Musicians and tone-language speakers share enhanced brainstem encoding but not perceptual benefits for musical pitch. Brain and Cognition, 77, 1-10. https://doi.org/10.1016/j.bandc.2011.07.006.

Bostwick, J., Seror, G. A., \& Neill, W. T. (2018). Tonality without structure. Music Perception: An Interdisciplinary Journal, 36(2), 243-249. https://doi.org/10.1525/MP.2018.36.2.243.

Brattico, E., Tervaniemi, M., Näätänen, R., \& Peretz, I. (2006). Musical scale properties are automatically processed in the human auditory cortex. Brain Research, 1117, 162-174. https://doi.org/10.1016/j.brainres.2006.08.023.

Brunet, D., Murray, M. M., \& Michel, C. M. (2011). Spatiotemporal analysis of multichannel EEG: CARTOOL. Computational Intelligence and Neuroscience, 2011, 813870. https://doi.org/10.1155/2011/813870. 
Chin, T., \& Rickard, N. S. (2012). The music USE (MUSE) questionnaire: An instrument to measure engagement in music. Music Perception: An Interdisciplinary Journal, 29, 429-446. https://doi.org/10.1525/mp.2012.29.4.429.

Cook, N. D., Fujisawa, T. X., \& Takami, K. (2006). Evaluation of the affective valence of speech using pitch substructure. IEEE Transactions on Audio, Speech and Language Processing, 14, 142-151. https://doi.org/10.1109/TSA.2005.854115.

Dalla Bella, S., Peretz, I., Rousseau, L., \& Gosselin, N. (2001). A developmental study of the affective value of tempo and mode in music. Cognition, 80(3), B1-B10. https://doi.org/10.1016/S0010-0277(00)00136-0.

Delorme, A., \& Makeig, S. (2004). EEGLAB: an open source toolbox for analysis of single-trial EEG dynamics including independent component analysis. Journal of Neuroscience Methods, 134, 9-21. https://doi.org/10.1016/j.jneumeth.2003.10.009,

Gandour, J., Dzemidzic, M., Wong, D., Lowe, M., Tong, Y., Hsieh, L., ... Lurito, J. (2003). Temporal integration of speech prosody is shaped by language experience: An fMRI study. Brain and Language, 84, 318-336. https://doi.org/10.1016/S0093-934X(02)00505-9.

Grosjean, F. (2001). The right of the deaf child to grow up bilingual. Sign Language Studies, 1(2), 110-114. https://doi.org/10.1353/sls.2001.0003.

Hailstone, J. C., Omar, R., Henley, S. M. D., Frost, C., Kenward, M. G., \& Warren, J. D. (2009). It's not what you play, it's how you play it: timbre affects perception of emotion in music. Quarterly Journal of Experimental Psychology (2006), 62, 2141-2155. https://doi.org/10.1080/17470210902765957.

Halpern, A. R., Martin, J. S., \& Reed, T. D. (2008). An ERP study of major-minor classification in melodies. Music Perception: An Interdisciplinary Journal, 25, 181-191. https://doi.org/10.1525/mp.2008.25.3.181.

Hamburger, H. L., \& v.d. Burgt, M. A. G. (1991). Global field power measurement versus classical method in the determination of the latency of evoked potential components. Brain Topography, 3, 391-396. https://doi.org/10.1007/BF01129642. 
Huron, D., \& Davis, M. (2013). The harmonic minor scale provides an optimum way of reducing average melodic interval size, consistent with sad affect cues. Empirical Musicology Review, 7(3-4), 103. https://doi.org/10.18061/emr.v7i3-4.3732.

Huron, D., Yim, G., \& Chordia, P. (Eds.). (2010). The effect of pitch exposure on sadness judgments: An association between sadness and lower than normal pitch. Retrieved from http://fliphtml5.com/bbwx/rxio/basic

Jasper, H. H. (1958). The 10-20 electrode system of the international federation. Electroencephalography and Clinical Neurophysiology, 10, 371-375.

Laurier, C., Lartillot, O., Eerola, T., \& Toiviainen, P. (2009). Exploring relationships between audio features and emotion in music. In ESCOM 2009: 7th triennial conference of european society for the cognitive sciences of music.

Liu, L., \& Kager, R. (2018). Monolingual and bilingual infants' ability to use non-native tone for word learning deteriorates by the second year after birth. Frontiers in Psychology, 9, 117. https://doi.org/10.3389/fpsyg.2018.00117.

Lopez-Calderon, J., \& Luck, S. J. (2014). ERPLAB: an open-source toolbox for the analysis of event-related potentials. Frontiers in Human Neuroscience, 8, 213. https://doi.org/10.3389/fnhum.2014.00213.

Losorelli, S., Nguyen, D. T., Dmochowski, J. P., \& Kaneshiro, B. (2017). NMED-T: A tempo-focused dataset of cortical and behavioral responses to naturalistic music. In ISMIR (pp. 339-346).

Magne, C., Schön, D., \& Besson, M. (2006). Musician children detect pitch violations in both music and language better than nonmusician children: Behavioral and electrophysiological approaches. Journal of Cognitive Neuroscience, 18(2), 199-211. https://doi.org/10.1162/jocn.2006.18.2.199.

Park, M., Gutyrchik, E., Welker, L., Carl, P., Pöppel, E., Zaytseva, Y., ... Bao, Y. (2014). Sadness is unique: neural processing of emotions in speech prosody in musicians and non-musicians. Frontiers in Human Neuroscience, 8, 1049. https://doi.org/10.3389/fnhum.2014.01049.

Parncutt, R. (2014). The emotional connotations of major versus minor tonality: One or 
more origins? Musicae Scientiae, 18, 324-353.

https://doi.org/10.1177/1029864914542842.

Patil, K., Pressnitzer, D., Shamma, S., \& Elhilali, M. (2012). Music in our ears: the biological bases of musical timbre perception. PLoS Computational Biology, 8(11), e1002759. https://doi.org/10.1371/journal.pcbi.1002759.

Pearce, M. T., \& Wiggins, G. A. (2012). Auditory expectation: the information dynamics of music perception and cognition. Topics in Cognitive Science, 4, 625-652. https://doi.org/10.1111/j.1756-8765.2012.01214.x.

Polich, J. (1986). Attention, probability, and task demands as determinants of P300 latency from auditory stimuli. Electroencephalography and Clinical Neurophysiology, 63(3), 251-259. https://doi.org/10.1016/0013-4694(86)90093-3.

Polich, J. (1987). Response mode and P300 from auditory stimuli. Biological Psychology, 25, 61-71. https://doi.org/10.1016/0301-0511(87)90067-6.

Polich, J. (1997). On the relationship between EEG and P300: individual differences, aging, and ultradian rhythms. International Journal of Psychophysiology, 26, 299-317. https://doi.org/10.1016/S0167-8760(97)00772-1.

R Core Team. (2019). R: A language and environment for statistical computing. Vienna, Austria. Retrieved from https://www.R-project.org/

Santosa, H., Hong, M. J., \& Hong, K.-S. (2014). Lateralization of music processing with noises in the auditory cortex: an fNIRS study. Frontiers in Behavioral Neuroscience, 8, 418. https://doi.org/https://doi.org/10.3389/fnbeh.2014.00418.

Shahin, A., Bosnyak, D. J., Trainor, L. J., \& Roberts, L. E. (2003). Enhancement of neuroplastic P2 and N1c auditory evoked potentials in musicians. The Journal of Neuroscience, 23, 5545-5552. https://doi.org/10.1523/JNEUROSCI.23-13-05545.2003.

Skrandies, W. (1990). Global field power and topographic similarity. Brain Topography, 3, 137-141. https://doi.org/10.1007/BF01128870.

Stevens, C. J., Keller, P. E., \& Tyler, M. D. (2013). Tonal language background and detecting pitch contour in spoken and musical items. Psychology of Music, 41, 59-74. 
https://doi.org/10.1177/0305735611415749.

Stober, S., Cameron, D. J., \& Grahn, J. A. (2014). Using convolutional neural networks to recognize rhythm stimuli from electroencephalography recordings. In Advances in neural information processing systems (pp. 1449-1457).

Swaminathan, S., \& Schellenberg, E. G. (2015). Current emotion research in music psychology. Emotion Review, 7(2), 189-197. https://doi.org/10.1177/1754073914558282.

The MathWorks, Inc. (2019). MATLAB and statistics toolbox R2018b. Massachusetts, United States.

Tramo, M. J., Cariani, P. A., Delgutte, B., \& Braida, L. D. (2001). Neurobiological foundations for the theory of harmony in western tonal music. Annals of the New York Academy of Sciences, 930, 92-116. https://doi.org/10.1111/j.1749-6632.2001.tb05727.x.

Treder, M. S., Purwins, H., Miklody, D., Sturm, I., \& Blankertz, B. (2014). Decoding auditory attention to instruments in polyphonic music using single-trial EEG classification. Journal of Neural Engineering, 11, 026009. https://doi.org/10.1088/1741-2560/11/2/026009.

Tremblay, K. L., Ross, B., Inoue, K., McClannahan, K., \& Collet, G. (2014). Is the auditory evoked P2 response a biomarker of learning? Frontiers in Systems Neuroscience, 8, 28. https://doi.org/10.3389/fnsys.2014.00028.

Vaissière, J. (1983). Language-independent prosodic features. In W. J. M. Levelt, A. Cutler, \& D. R. Ladd (Eds.), Prosody: Models and measurements (Vol. 14, pp. 53-66). Berlin, Heidelberg: Springer Berlin Heidelberg. https://doi.org/10.1007/978-3-642-69103-4_5.

Winkler, I., Denham, S., \& Escera, C. (2013). Auditory event-related potentials. In D. Jaeger \& R. Jung (Eds.), Encyclopedia of computational neuroscience: Auditory event-related potentials (Vol. 27, pp. 1-29). New York, NY: Springer New York. https://doi.org/10.1007/978-1-4614-7320-6_99-1.

Yip, M. (2002). Tone. Cambridge University Press. 
https://doi.org/10.1017/CB09781139164559. 


\section{Appendix A}

List of All Languages Spoken by Participants

$\begin{array}{cc}\text { Language } & \text { Category } \\ \text { Albanian } & \text { Intonation } \\ \text { Arabic } & \text { Intonation } \\ \text { Balochi } & \text { Intonation } \\ \text { Bengali } & \text { Intonation } \\ \text { Bulgarian } & \text { Intonation } \\ \text { Burmese } & \text { Tonal } \\ \text { Cantonese } & \text { Tonal } \\ \text { Cebuano } & \text { Intonation } \\ \text { Mandarin } & \text { Tonal } \\ \text { English } & \text { Intonation } \\ \text { Farsi } & \text { Intonation } \\ \text { Finnish } & \text { Intonation } \\ \text { French } & \text { Intonation } \\ \text { Georgian } & \text { Intonation } \\ \text { German } & \text { Intonation } \\ \text { Hindi } & \text { Intonation } \\ \text { Italian } & \text { Intonation } \\ \text { Kanada } & \text { Intonation } \\ \text { Kyrgyz } & \text { Intonation } \\ \text { Macedonian } & \text { Intonation } \\ \text { Marathi } & \text { Intonation } \\ \text { Nepalese } & \text { Intonation } \\ \text { Pulaar } & \text { Intonation } \\ \text { Punjabi } & \text { Intonal } \\ \text { Romanian } & \text { Intonation } \\ \text { Sparaeki } & \text { Intonation } \\ \text { Intonation } \\ \text { Intonation } \\ \text { Intonation } \\ \text { Intonation } \\ \text { Inton }\end{array}$




\section{Appendix B}

Language and Musical Experience Questionnaire

1. What is/are your native language(s)?

2. What other language(s) do you speak? (please include the level of knowledge OR the years of experience you have in each language)

3. On average, how often do you listen to music in a week?

$\square$ Less than once a week

$\square \quad$ 1-2 times a week

3-4 times a week

$\square$ 5-6 times a week

More than 6 times a week

4. On average, how many hours do you purposely listen to music a day (as opposed to music in the environment that you have no control over, e.g., music in cafes, stores)?
$\square$ Less than 1 hour per day
$\square \quad 1-2$ hours per day
3-4 hours per day
$\square$ 5-6 hours per day
$\square$ More than 6 hours per day

5. Have you played/do you play a music instrument (includes singing, practice, and performance)?

$\square \quad$ No (you may skip the remaining questions)

$\square$ Yes, I've played (name of music instrument) for years (please continue on to question 6)

6. At the peak of your interest, how many hours per day did you play/practice the music instrument (includes singing)?

I played/practiced hours per day (please fill in the hours of practice)

7. How long since you last regularly played a music instrument (includes singing, practice, and performance)?

$\square \quad$ Less than a week ago

$\square$ Less than a month ago

$\square$ Less than 1 year ago

$\square$ Between 1 and 5 years ago

$\square$ Between 5 and 10 years ago

$\square$ More than 10 years ago 


\section{Statutory Declaration}

\begin{tabular}{|l|l|}
\hline Family Name, Given/First Name & Zhao, Shuqing \\
\hline Matriculation number & 30000632 \\
\hline $\begin{array}{l}\text { What kind of thesis are you submitting: } \\
\text { Bachelor-, Master- or PhD-Thesis }\end{array}$ & Bachelor Thesis \\
\hline
\end{tabular}

English: Declaration of Authorship

I hereby declare that the thesis submitted was created and written solely by myself without any external support. Any sources, direct or indirect, are marked as such. I am aware of the fact that the contents of the thesis in digital form may be revised with regard to usage of unauthorized aid as well as whether the whole or parts of it may be identified as plagiarism. I do agree my work to be entered into a database for it to be compared with existing sources, where it will remain in order to enable further comparisons with future theses. This does not grant any rights of reproduction and usage, however.

This document was neither presented to any other examination board nor has it been published.

\section{German: Erklärung der Autorenschaft (Urheberschaft)}

Ich erkläre hiermit, dass die vorliegende Arbeit ohne fremde Hilfe ausschließlich von mir erstellt und geschrieben worden ist. Jedwede verwendeten Quellen, direkter oder indirekter Art, sind als solche kenntlich gemacht worden. Mir ist die Tatsache bewusst, dass der Inhalt der Thesis in digitaler Form geprüft werden kann im Hinblick darauf, ob es sich ganz oder in Teilen um ein Plagiat handelt. Ich bin damit einverstanden, dass meine Arbeit in einer Datenbank eingegeben werden kann, um mit bereits bestehenden Quellen verglichen zu werden und dort auch verbleibt, um mit zukünftigen Arbeiten verglichen werden zu können. Dies berechtigt jedoch nicht zur Verwendung oder Vervielfältigung.

Diese Arbeit wurde noch keiner anderen Prüfungsbehörde vorgelegt noch wurde sie bisher veröffentlicht.

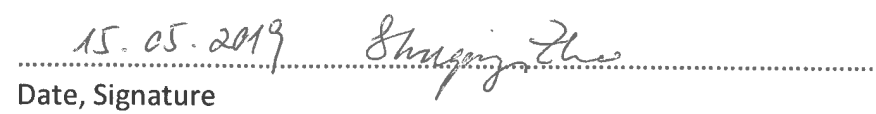

